\title{
Polarization Possibilities of Small Spin-Orbit Interaction in Strained-Superlattice Photocathodes
}

\author{
T. Maruyama, A. Brachmann, J.E. Clendenin, E.L. Garwin, \\ K. Ioakeimidi, and R.E. Kirby \\ SLAC National Accelerator Laboratory, Menlo Park, California 94025, USA \\ R. Prepost \\ Department of Physics, University of Wisconsin, Wisconsin 53706, USA
}

A.M. Moy

SVT Associates, Eden Prairie, Minnesota 55344, USA

\begin{abstract}
Strained-superlattice photocathodes based on InGaP/GaAs were investigated. The photocathode performance is found highly dependent on the superlattice parameters. The electron confinement energy in superlattice appears important.
\end{abstract}

Keywords: polarized electrons, superlattice

PACS: 72.25.Fe, 73.21.Cd, 79.60.-i

\section{INTRODUCTION}

The strained-superlattice structure based on GaAsP/GaAs, with a maximum polarization as high as $90 \%$ and more than $1 \%$ quantum efficiency, is presently the prime candidate for the ILC polarized electron photocathodes. A recent systematic study shows, however, that the peak polarization seems saturated even though the heavy-hole (HH) and light-hole ( $\mathrm{LH})$ band splitting is increased significantly, indicating that there is a material specific spin relaxation mechanism [1]. It is widely accepted that the D'yakonov-Perel mechanism is the dominant spin relaxation mechanism in the III-V compound superlattice structures with a low $p$-doping $\left(\leq 10^{17}\right.$ $\mathrm{cm}^{-3}$ ), and that the spin relaxation may be reduced by choosing a material with a smaller spin-orbit interaction. As the spin-orbit interaction in phosphides is much smaller than in arsenides, strained-superlattice structure based on $\mathrm{InGaP} / \mathrm{GaAs}$ were investigated. The computer code SPECCODE developed by Subashiev and Gerchikov has been used for calculating the band structures in superlattice [2]. 


\section{STRAIN EFFECT IN GaAsP/GaAs SUPERLATTICE}

The lattice-mismatch between the well (GaAs) and the barrier ( GaAsP) changes when the phosphorus faction is varied. While a larger phosphorus fraction generates a larger strain and therefore a larger energy splitting between the $\mathrm{HH}$ and $\mathrm{LH}$ bands, the strain within a layer may relax if the lattice-mismatch becomes too large. The phosphorus fraction was increased from 0.25 to 0.40 keeping the total superlattice thickness constant. Figure 1 shows the peak polarization as a function of the phosphorus fraction. The measured HH-LH energy splitting is also shown in the figure together with the SPECCODE predictions for $100 \%$ strained structure. As the phosphorus fraction is increased, the layer begins to relax. But the relaxation does not exceed $16 \%$ even at the highest phosphorus fraction of 0.4. Although the HH-LH energy splitting increases from $60 \mathrm{meV}$ to $89 \mathrm{meV}$, the peak polarization does not change significantly at about $85 \%$, indicating that this degree of energy splitting is sufficient to maximize the spin polarization. A spin-relaxation mechanism specific to the GaAsP/GaAs structure appears to be present.

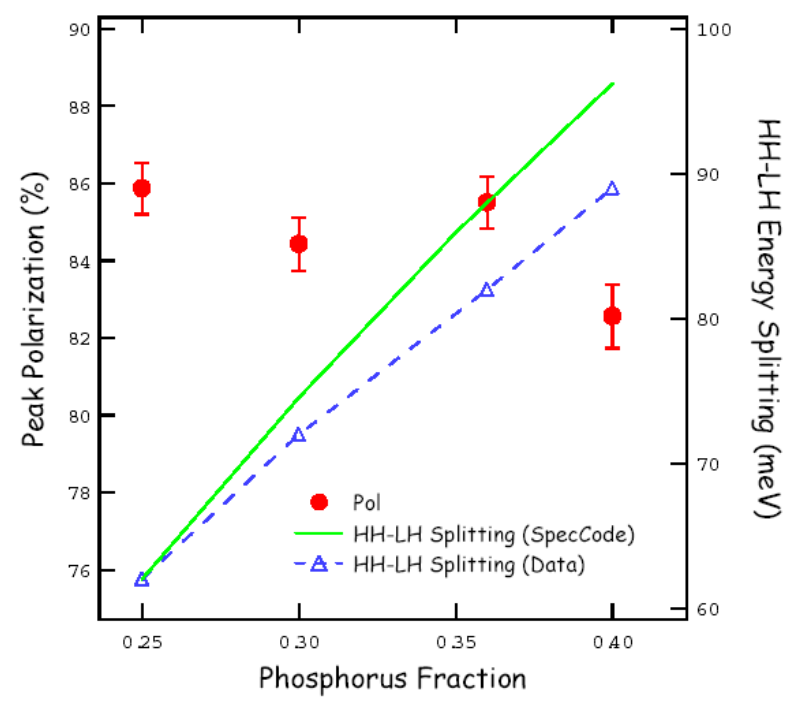

FIGURE 1. Peak polarization (solid circles) and measured HH-LH splitting (triangles) as a function of the phosphorus fraction. The HH-LH splitting calculated by SPECCODE for fully strained structure is shown in solid line.

\section{SPIN-RELAXATION AND InGaP/GaAs SUPERLATTICE}

In photoemission from a thin epitaxial layer, the polarization, $P$, may be expressed by

$$
P=P_{0} \frac{\tau_{s}}{\tau_{s}+<\tau>} P_{B B R},
$$


where $P_{0}$ is the initial polarization, $\tau_{s}$ the spin-relaxation time, $\langle\tau\rangle$ the average photoemission time, and $P_{B B R}$ any additional depolarization generated in the band bending region. The average photoemission time for a 100-nm thick strained GaAs cathode has been measured to be $\langle\tau\rangle \sim 3$ ps [3]. A spin-relaxation time shorter than about 50 ps would have a significant effect on polarization. The dominant spin relaxation mechanism in the III-V compound superlattice structures is the D'yakonov-Perel mechanism via the spin-orbit interaction. Therefore, the spin relaxation may be reduced by choosing a material with a smaller spin-orbit interaction. As the spin-orbit interaction in phosphides is much smaller than in arsenides, we have investigated the strained-superlattice structure based on InGaP/GaAs, replacing GaAsP with InGaP.

As $\mathrm{In}_{0.48} \mathrm{Ga}_{0.52} \mathrm{P}$ is lattice-matched to $\mathrm{GaAs}$, it is possible to grow a strained-well superlattice using less than $48 \%$ In or a strained-barrier superlattice using more than $48 \%$ In. Three different structures have been grown at SVT Associates using gassource MBE: one strained-well $\mathrm{In}_{0.32} \mathrm{Ga}_{0.68} \mathrm{P} / \mathrm{GaAs}$ structure and two strained-barrier $\mathrm{In}_{0.65} \mathrm{Ga}_{0.35} \mathrm{P} / \mathrm{GaAs}$ structures. A lattice-mismatch of $1.25 \%$ between $\mathrm{GaAs}$ and $\mathrm{InGaP}$ is used for both structures. Table I summarizes the sample parameters together with the experimental results on quantum efficiency $(\mathrm{QE})$ at $670 \mathrm{~nm}$ and peak polarization.

Table I. Sample parameters and experimental results

\begin{tabular}{|l|l|l|l|l|l|l|l|l|}
\hline & $\begin{array}{l}\text { GaAs } \\
(\mathrm{nm})\end{array}$ & $\begin{array}{l}\text { InGaP } \\
(\mathrm{nm})\end{array}$ & In & $\begin{array}{l}\text { Band Gap } \\
\text { Energy }(\mathrm{eV})\end{array}$ & $\begin{array}{l}\text { HH-LH Splitting } \\
(\mathrm{meV})\end{array}$ & $\begin{array}{l}\Delta \mathrm{E}_{1 \mathrm{e}} \\
(\mathrm{meV})\end{array}$ & $\begin{array}{l}\text { QE (\%) } \\
670 \mathrm{~nm}\end{array}$ & $\begin{array}{l}\text { Polarization } \\
(\%)\end{array}$ \\
\hline 1 & 4.0 & 4.0 & 0.32 & 1.57 & 108 & 76 & 0.4 & 68 \\
\hline 2 & 1.5 & 4.0 & 0.65 & 1.47 & 94 & 43 & 0.002 & 40 \\
\hline 3 & 4.0 & 1.5 & 0.65 & 1.44 & 54 & 17 & 0.01 & 68 \\
\hline
\end{tabular}

\section{DISCUSSION}

As seen in Table I, the photocathode performance is highly dependent on the superlattice parameters. Sample 1, the strained-well structure, yielded $68 \%$ polarization, while the $\mathrm{GaAs}_{0.64} \mathrm{P}_{0.36} / \mathrm{GaAs}$ structure with the same superlattice parameters shows $86 \%$ as seen in Figure 1. In particular, the two strained-barrier samples yielded very different QE and polarization. While Sample 2 was expected to have a higher polarization than Sample 3 because of the larger HH-LH splitting, the experimental result was opposite, indicating that the superlattice parameters (well and barrier thickness) are more important than having a large HH-LH energy splitting.

Spin relaxation near room temperature is dominated by a spin precession mechanism in an internal crystal magnetic field, the D'yakonov-Perel mechanism. The spin relaxation rate depends on the effective magnetic field, which results from the lack of crystal inversion symmetry and the spin-orbit coupling, and is given by [4] 


$$
\frac{1}{\tau_{s}}=\frac{16 \mathrm{k}_{\mathrm{B}} \mathrm{T}\left(m^{*}\right)^{3}\left(\gamma \Delta \mathrm{E}_{\mathrm{le}}\right)^{2} \tau_{p}}{\hbar^{8}}
$$

where $\gamma$ is a material-specific parameter related to the spin splitting of the conduction band and is proportional to the spin-orbit splitting, $\Delta E_{l e}$, the electron confinement energy (ECE), $m^{*}$ the electron effective mass, and $\tau_{p}$ the momentum relaxation time. Eq. (2) shows how the spin relaxation rate depends on the spin-orbit interaction $(\gamma)$ and the electron confinement energy. The ECE value for the three structures calculated by SPECCODE is given in Table I, and the ECE for the $\mathrm{GaAs}_{0.64} \mathrm{P}_{0.36} / \mathrm{GaAs}$ structure is 49 $\mathrm{meV}$. Sample 1 has a factor of 1.6 larger ECE than the reference strained-well $\mathrm{GaAs}_{0.64} \mathrm{P}_{0.36} / \mathrm{GaAs}$ structure. Comparing the two strained-barrier samples, Sample 2 has a factor of 2.5 larger ECE than Sample 3, resulting in a factor of 6 larger spin relaxation rate according to Eq. (2). In a superlattice structure with a larger ECE, the electrons will scatter more at the barriers, resulting in a spin depolarization and a lower vertical transport probability and therefore a lower QE. Furthermore, because of the scatterings the average photoemission time may be much longer than 3 ps, becoming more susceptible to spin depolarization. Aulenbacher et al. reported a significantly longer photoemission time for an InAlGaAs/AlGaAs superlattice structure, suggesting that the superlattice barrier layers are responsible [5].

\section{CONCLUSIONS}

In an attempt at reducing the spin relaxation in superlattice, InGaP/GaAs strainedsuperlattice structures were investigated. The photocathode performance is found dependent on the superlattice parameters. Especially the electron confinement energy appears very important.

\section{ACKNOWLEDGMENTS}

This work was supported by US Department of Energy Small Business Innovation Research Grant No. DE-FG02-05ER84382 and Contract Nos. DE-AC03-76SF00515 (SLAC) and DE-AC02-76ER00881 (UW).

\section{REFERENCES}

1. T. Maruyama, D.-A. Luh, A. Brachmann, J.E. Clendenin, E.L. Garwin, S. Harvey, J. Jiang, R.E. Kirby, C.Y. Prescott, R. Prepost, and A.M. Moy, Appl. Phys. Letters 85, 2640 (2004).

2. A.V. Subashiev, L.G. Gerchikov and A.N. Ipatov, J. of Appl. Phys. 96, 1511 (2004).

3. K. Aulenbacher et al., J. of Appl. Phys. 92, 7536 (2002).

4. M.I. D’yakonov and V. Yu. Kachorovskii, Sov. Phys.-Semicond. 20110 (1986); A. Tackeuchi, O. Wada, and Y. Nishikawa, Appl. Phys. Letters 70, 1131 (1997).

5. Aulenbacher et al., Proceedings of the $16^{\text {th }}$ International Spin Physics Symposium and Workshop on Polarized Electron Sources and Polarimeters, Trieste, Italy, 10-16 October 2004. p. 922 


\title{
FINAL REPORT
}

\author{
for
}

Project entitled:

High polarization, superlattice cathodes

\section{Partner:}

SVT Associates

\section{DOE Laboratory:}

SLAC National Accelerator Laboratory

This final report is being submitted to meet the requirements in the CRADA agreement CRADA SLAC- $\quad 281$

As stated in the following paragraph.

ARTICLE XI: REPORTS AND ABSTRACTS

The Parties agree to produce the following deliverables: an initial abstract suitable for public release; and a final report to include a list of Subject Inventions. It is understood that the Contractor has the responsibility to provide this information at the time of its completion to the Contracting Officer and the DOE Office of Scientific and Technical Information.

Date submitted: 2010 APR 26

Submitted by: James E. Simpson 